\title{
LINEA 3.
}

GESTIÓN PÚBLICA 


\section{CAPITULO}

Impuesto Departamental al Consumo en Boyacá para el Año 2017 Respecto a Vigencia Fiscal Anterior Frente a la Aplicación de las Leyes 1801, 1816 Y 1819 de 2016

Departmental Tax on Consumption in Boyacá for the Year 2017 Regarding Previous Tax Validity Faced with the Application of Laws 1801, 1816 and 1819 of 2016

Elina Ulloa Sáenz Carlos Andrés Aranda Camacho Luis Rodrigo Ramírez Ahumada 


\section{Resumen}

Un grupo de grandes contribuyentes de impuesto al consumo en el Departamento de Boyacá manifiesta una significativa reducción en las ventas del primer trimestre del año 2017, señalan como causa la entrada en vigencia del Código Nacional de Policía y Convivencia Ciudadana "Para Vivir en Paz", la Ley 1819 de 2016 o Reforma Tributaria y a la Ley 1816 de 2016 o Ley de Licores. La Secretaría de Hacienda del Departamento con el fin de conocer el impacto social de los sectores comerciales en relación con el pago de los tributos departamen-tales, desarrolló un estudio de opinión en establecimientos comerciales con el fin de establecer el comportamiento de las ventas de cigarrillos, cervezas, vinos, licores y similares durante el primer trimestre del año 2017. Se encontró que para la mayoría de los comerciantes las ventas han disminuido significativamente y que señalan como causa las medidas del Código de Policía o de los Decretos Municipales que regulan las ventas de los mencionados productos.

Palabras Clave: Boyacá, Policía, Convivencia, Consumo.

\section{Abstract}

A group of the biggest taxpayers of consumption tax in the Department of Boyacá shows a significant reduction in sales in the first quarter of 2017, they point to the entry into force of the National Code of Police and Citizen's Coexistence to Live in Peace, the Law 1819 of 2016 or Tax Reform and Law 1816 of 2016 or Law of Liqueurs. The Secretary of Finance of the Department, with the purpose of knowing the social impact of the commercial sectors in relation to the payment of the departmental taxes, developed an opinion survey in commercial establishments in order to establish the behavior of sales of cigarettes, beers, wines, liqueurs and the like during the first quarter of 2017. It was found that for the majority of the merchant's sales have decreased significantly and that they indicate as causes the measures of the Police Code or of the Municipal Decrees that regulate the sales of the Products.

Key words: Boyacá, Police, comunal living, consumption.

\section{INTRODUCCIÓN}

El panorama comercial de los productos objeto de los tribu-tos por concepto de consumo en el Departamento de Boyacá es un tanto sombrío según la manifestado por los comer-ciantes entrevistados en Tunja, Duitama, Sogamoso, Paipa y Chiquinquirá.

En líneas generales, la abrumadora mayoría de los comerciantes entrevistados expresa haberse visto afectados por la entrada en vigencia del nuevo Código Nacional de Policía y por la Reforma Tributaria y la Ley de Licores o Ley 1816 de 2017, manifestada ésta última en el incremento del precio de los productos que, a su vez, se refleja en alzas del precio de venta al consumidor final.

Casi la mitad de los comerciantes entrevistados manifiesta que las ventas del año 2016 fueron buenas o muy buenas, en contraste con lo que manifiestan sobre las ventas en lo transcurrido de 2017, pues la gran mayoría señala que son regulares o muy malas.

En cuanto a la prospectiva de las ventas a final del 2017 el ánimo de los comerciantes del Departamento es bastante pesimista, pues poco más de la mitad expresan que las ventas serán peores que las del año pasado.

En lo relativo al Código Nacional de Policía una porción significativa de los entrevistados manifiesta que tiene medidas buenas, aunque los afectan en su actividad comercial y unos pocos dicen no haberse visto afectados. 
A continuación, se presenta el detalle de los hallazgos del estudio. Es importante tener en cuenta que el método de recolección fue una entrevista con un cuestionario semi-estructurado y que, se realizó la unificación de la amplia variedad de respuestas mediante la siguiente jerarquía de categorías, que constituyen el grueso de los objetivos del estudio y que, a su vez, determinaron el tipo de conclusiones a las que se ha llegado:

Ilustración 33. Hallazgos de la investigación

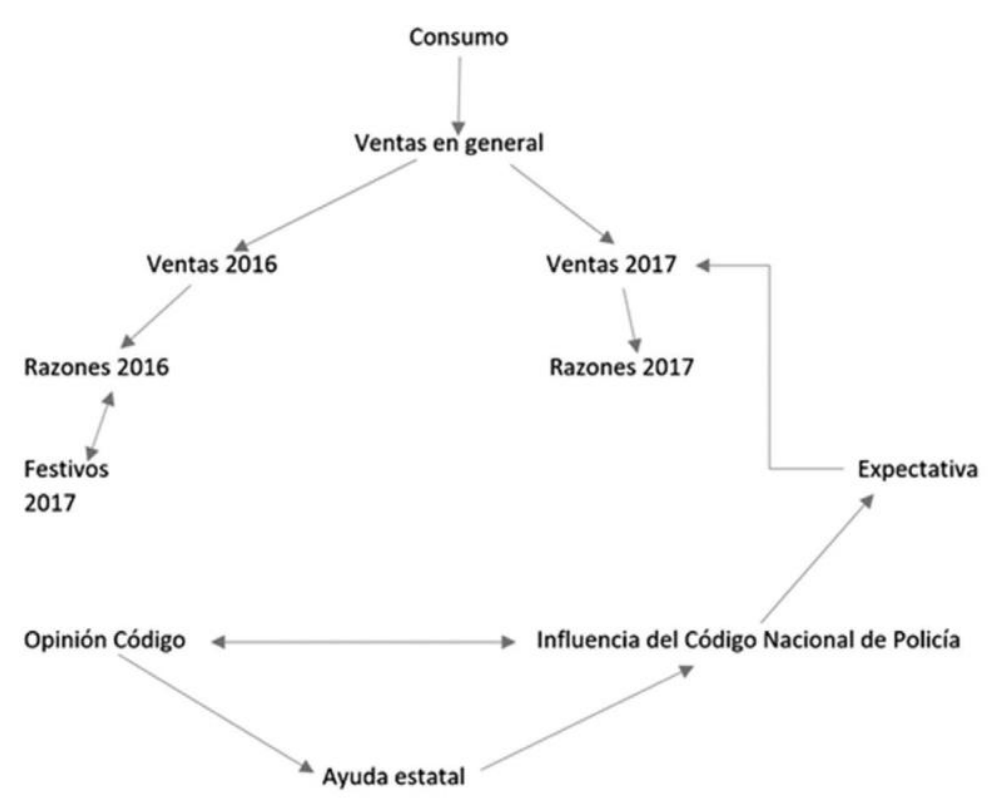

La gráfica permite evidenciar el nivel de importancia que esta problemática adquiere para la administración tributaria departamental, pues es de su interés incidir de manera apositiva en las ventas, lo que redundaría en un aumento del recaudo.

\section{Planteamiento del problema}

En concordancia con los objetivos del estudio se planteó la siguiente pregunta de investigación: ¿Cuáles son las causas que generan la disminución en el recaudo del impuesto al consumo en el Departamento de Boyacá para el año 2017 respecto a vigencias fiscales anteriores?. Trazando claramente cuatro momentos a investigar así: i) Analizar el impacto de la entrada en vigencia del Código Nacional de Policía y Convivencia "Para vivir en paz" (Ley 1081 de 2016) respecto al consumo de cigarrillos, cervezas, licores, vinos y similares en el Departamento de Boyacá. ii) Analizar el impacto de las Leyes 1816 de 2016 y 1819 de 2016 con relación a la carga tributaria de cervezas, licores, vinos aperitivos y similares y cigarrillos en el Departamento de Boyacá. iii) Realizar estudio de opinión sobre el comportamiento del consumo de cer-vezas, licores, vinos aperitivos y similares y cigarrillos en el Departamento de Boyacá en los 4 primeros meses del año 2017. iv) Determinar posibilidades de intervención por parte de la Secretaria de Hacienda de Boyacá respecto a las causas que impactan la disminución del recaudo de impuesto terri-torial al consumo.

Igualmente, a manera de hipótesis se afirmó que el recaudo por concepto de consumo durante los primeros tres meses fue bueno porque las medidas del Código Nacional de Policía fueron pedagógicas. Una vez se empezó con multas y cierres el recaudo por consumo disminuyó en la medida en que los establecimientos se vieron abocados a dar estricto cumplimiento a las medidas del Código. En el mismo sentido, el recaudo por consumo de cigarrillos, cervezas, licores, vinos y similares ha sido menor en los primeros meses del 2017 porque ha habido dos festivos y las celebraciones y, las vacaciones de Semana Santa ocurrieron en abril mientras que, en el 2016, hubo tres festivos y la Semana Santa se desarrolló en marzo. 


\section{OBJETIVOS}

\section{Objetivo general}

Conocer el impacto sentido por los comerciantes de vinos, licores, cervezas y cigarrillos del Departamento de Boyacá del Código de Policía y Convivencia ciudadana.

\section{Objetivos específicos}

- Recoger las opiniones de los comerciantes de vinos, cervezas, icores y cigarrillos sobre el Código de Policía y Convivencia Ciudadana.

- Indagar en el tipo y magnitud del impacto del Código de Policía en la dinámica comercial de licores, cervezas, vinos y cigarrillos en el Departamento de Boyacá.

- Comparar las ventas de licores, cervezas, vinos y cigarrillos en los primeros tres meses del año 2016 con el mismo período del 2017.

\section{HIPÓTESIS}

La entrada en vigencia del Código de Policía y Convivencia Ciudadana, la Ley 1816 de 2016 y la Ley 1819 de 2016 ha tenido un impacto negativo en las ventas de cervezas, vinos, licores y cigarrillos en el Departamento de Boyacá, lo que ha causado un impacto negativo en el recaudo de los tributos de que son objeto los mencionados productos.

\section{PREGUNTAS DE INVESTIGACIÓN}

¿Cuál ha sido el impacto de la entrada en vigencia del Código de Policía en las ventas de cervezas, licores, vinos y cigarrillos en el Departamento de Boyacá?
¿Qué opinan los comerciantes de cervezas, vinos, licores y cigarrillos del Código de Policía?

$¿$ Hay diferencias en el impacto en las ventas de cervezas, licores, vinos y cigarrillos en las diferentes ciudades de Boyacá?

\section{JUSTIFICACIÓN}

Dentro de las naturaleza y funciones del Estado y de las Administraciones Públicas se encuentra la de recaudar fondos de sus afiliados para su funcionamiento. El Departamento de Boyacá, asignó estas funciones a la Secretaría de Hacienda, específicamente a la Dirección de Recaudo y Fiscalización para vigilar el cumplimiento tributario en las ventas de productos de consumo tales como cervezas, vinos, licores y similares y cigarrillos. Para el Departamento de Boyacá el recaudo por estos conceptos constituye una de las más grandes fuentes de financiamiento para el desarrollo del Departamento, por esto tiene vital importancia para la administración tributaria departamental conocer de primer mano el impacto que las medidas del orden nacional puedan tener en el consumo de talles productos en su jurisdicción. La razón de este estudio no es otra que determinar el tipo y calidad de impacto que la entrada en vigencia de las medidas que regulan el consumo de licores, vinos, cervezas y cigarrillos puedan tener en las ventas $y$, por ende, en el recaudo tributario por el mismo concepto.

\section{MARCO NORMATIVO DEL IMPUESTO AL CONSUMO}

\section{Ley 1816 de 2016}

Conocida como Ley de Licores, la Ley 1816 de 2016 tiene especifica que el objeto del monopolio como arbitrio rentístico 
sobre los licores destilados es el de obtener recursos para los departamentos, con una finalidad social asociada a la financiación preferente de los servicios de educación y salud y al de garantizar la protección de la salud pública.

\section{Ley 1819 de 2016}

Se le conoce como Reforma Tributaria, por medio de la cual se adopta una reforma tributaria estructural, se fortalecen los mecanismos para la lucha contra la evasión y la elusión fiscal, y se dictan otras disposiciones.

\section{Aspectos Metodológicos}

\section{Diseño metodológico}

El desarrollo de este estudio tiene como fin último contrastar los aspectos de la normatividad mencionada anteriormente y las opiniones de los vendedores de cigarrillos, licores, vinos, cervezas y similares en cuanto al impacto de dichas medidas en las ventas los productos. Mediante un estudio de opinión se recogieron las percepciones, inquietudes y creencias de los vendedores de los mencionados productos, teniendo en cuenta factores como tamaño y tipo del negocio, ubicación y ciudad, para enmarcarlas dentro de las normas que pudieran afectar el consumo y así poder tener una visión panorámica de la problemática de la disminución del recaudo por concep-to de impuesto al consumo en los primeros cuatro meses en el Departamento de Boyacá.

\section{Recolección y análisis de la información}

Se plantea realizar un trabajo de investigación en dos vías: una documental y una de recolección de información que, al confrontarlas permitan establecer un marco de certeza en cuanto a las razones que explican la disminución en el recaudo del impuesto al consumo.

\section{Documental}

Identificación y análisis de los posibles impactos de la normatividad que aplica de manera directa al problema formu-lado con relación Ley 1816 de 2016 y 1819 de 2016 y Código Nacional de Policía y Convivencia "Para vivir en paz".

\section{Recolección de información}

Se realizaron 100 entrevistas a propietarios de establecimientos públicos como tiendas, bares, discotecas, supermercados y grandes superficies, respecto al impacto de la medidas normativas en cuanto a las ventas en Tunja, Paipa, Duitama, Sogamoso y Chiquinquirá, basados en un criterio geográfico y poblacional conforme a la disposición de los operativos de control rentístico de la Dirección de Recaudo y Fiscalización de la Secretaría de Hacienda de Boyacá.

\section{Tabla 16. Muestra}

\begin{tabular}{ll}
\multicolumn{1}{r}{ Municipio } & \multicolumn{1}{c}{ Tamaño de la Muestra Seleccionada } \\
\hline Tunja & 30 \\
\hline Sogamoso & 20 \\
\hline Duitama & 20 \\
\hline Paipa & 15 \\
\hline Chiquinquirá & 15 \\
\hline Total & 100 \\
\hline
\end{tabular}

El tamaño de la muestra seleccionada obedece en primer lugar a que en estos Municipios se encuentra el $60 \%$ de los habitantes del Departamento, concentrando, a su vez, la mayor parte de los consumidores. La selección de los establecimientos se dio en función de los resultados de los operativos de control rentístico realizados por la Dirección de Recaudo y Fiscalización. La escogencia de estos establecimientos 
facilitará el acceso a información de primera mano en cuanto a ventas, factores que influyen en las mismas, conocimiento de medidas restrictivas y correctivas en el Código de Policía, comparación entre las ventas de años anteriores y el presente año, incidencia de festivos y Semana Santa en las ventas, impacto de la Ley 1819 de 2016 y medidas que la Administración Tributaria Departamental podría tomar para favorecer el consumo y, por ende, el recaudo.

Instrumentos de recolección: Entrevista semi estructurada para conocer el impacto sentido de la medidas normativas en cuanto a las ventas en establecimientos públicos (Tiendas, Bares, Discotecas y supermercados) en Tunja, Paipa, Duitama, Sogamoso y Chiquinquirá.

Temas de la entrevista

- Percepción de las ventas en 2016 y 2017.

- Código Nacional de Policía y Convivencia "Para vivir en paz": Conocimiento, opiniones, medidas, impacto.

- Leyes 1816 y 1819 de 2106: impacto en las ventas.

- Medidas de apoyo del Departamento para mejorar las ventas de cigarrillos, cervezas, licores, vinos y similares.

\section{ANÁLISIS Y DISCUSIÓN DE RESULTADOS}

Tabla 17. Apreciación general de las ventas por cuidad

\begin{tabular}{lrrrrr}
$\begin{array}{l}\text { Cuenta de 1. Apreciación ventas } \\
\text { Etiquetas de fila }\end{array}$ & $\begin{array}{l}\text { Etiquet: } \\
\text { BUENAS }\end{array}$ & & & & \\
CHIQUINQUIRÁ & 8 & & 1 & 6 & 15 \\
DUITAMA & 7 & & 2 & 11 & 20 \\
PAIPA & 3 & & 5 & 7 & 15 \\
SOGAMOSO & 3 & 1 & 3 & 13 & 20 \\
TUNJA & 10 & 1 & 5 & 14 & 30 \\
\hline Total general & $\mathbf{3 1}$ & $\mathbf{2}$ & $\mathbf{1 6}$ & $\mathbf{5 1}$ & $\mathbf{1 0 0}$ \\
\hline
\end{tabular}

En cuanto a la apreciación general de las ventas se encontró que la mayoría de los entrevistados manifiestan que son regulares o muy malas, destacándose que 51 dicen que son regulares. Poco más de 30 expresan que las ventas son buenas o muy buenas. Por tratarse de una apreciación general ésta distribución de respuestas debe tomarse con beneficio de duda, sobretodo porque la información fue brindada directamente a contratistas de la Dirección de Recaudo y Fiscalización y, para nadie es un secreto, que culturalmente existe cierto grado de rechazo a brindar información sensible a los entes del Estado.

Tabla 18. Apreciación de ventas por mejores meses de 2016

\begin{tabular}{|c|c|c|c|c|c|}
\hline \multicolumn{2}{|c|}{$\begin{array}{l}\text { Cuenta de 1. Apreciación ventas Etiquetas de columna } \mathbf{V} \\
\text { Etiquetas de fila }\end{array}$} & \multicolumn{4}{|c|}{ MUY BUENAS MUY MALAS REGULAR Total general } \\
\hline ENERO & 3 & 3 & 1 & 5 & 9 \\
\hline MARZO & & 1 & 1 & 2 & 4 \\
\hline ABRIL & & & 1 & & 1 \\
\hline MAYO & & & 1 & & 1 \\
\hline JUNIO & & 1 & 3 & 4 & 16 \\
\hline JULо & 1 & 1 & 1 & 2 & 4 \\
\hline AGOSTO & 1 & 1 & & & 1 \\
\hline SEPTIEMBRE & 1 & & 1 & 6 & 8 \\
\hline OCTUBRE & 1 & 1 & 1 & & 2 \\
\hline NOVIEMBRE & & 3 & 1 & 2 & 6 \\
\hline DICIEMBRE & 5 & 1 & & 8 & 14 \\
\hline ENERO & & & & 1 & 1 \\
\hline NINGUNO & 2 & 2 & 3 & 5 & 10 \\
\hline NO HUBO MES BUENO & & & & 1 & 1 \\
\hline TODO EL AÑO & 4 & 4 & & 6 & 10 \\
\hline (en blanco) & 1 & 1 & 2 & 9 & 12 \\
\hline Total general & 31 & 2 & 16 & 51 & 100 \\
\hline
\end{tabular}

Se destaca que para la mayoría de entrevistados el mejor mes para sus ventas fue junio, seguido por diciembre, curiosamente coincidiendo con temporadas de vacaciones de los estudiantes universitarios, quienes según manifestaron va-rios entrevistados, se cuentan dentro de sus mejores clientes. Parece que las opiniones están divididas pues otro segmento importante también manifestó que los peores meses paras las ventas fueron junio, septiembre y diciembre. Hay que tener en cuenta que en junio el Código de Policía no había entrado en vigencia pero que en algunos de los municipios entrevistados sí había un Decreto Municipal que ya regulaba varias de las conductas asociadas a la venta y consumo de 
cigarrillos, cervezas, licores, vinos y similares, por lo cual la entrada en vigencia del Código podría no haber afectado en mayor medida las ventas del año pasado.

Tabla 19 Apreciación de ventas 2017 por ciudad

\begin{tabular}{|c|c|c|c|c|}
\hline \\
\hline \multicolumn{5}{|c|}{ Etiquetas de fila } \\
\hline CHIQUINQUIRÁ & 3 & 5 & 6 & 14 \\
\hline DUITAMA & 3 & 6 & 11 & 20 \\
\hline PAIPA & 2 & 4 & 9 & 15 \\
\hline SOGAMOSO & 4 & 4 & 12 & 20 \\
\hline TUNJA & 4 & 9 & 17 & 30 \\
\hline Total general & 16 & 28 & 55 & 99 \\
\hline
\end{tabular}

En lo relativo a la apreciación de las ventas para lo que corri-do del presente año por ciudad se evidencia que apenas 16 de los 100 entrevistados manifestó que son buenas, incluso, ninguno expresó que muy buenas. Por el contrario, la amplia mayoría de los entrevistados se mostró bastante pesimista, pues 55 dijeron que las ventas del 2017 hasta ahora han sido regulares y 28 dijeron que muy malas. Se destaca la cifra de Paipa, donde 13 de los 15 entrevistados expresaron su des-contento con las ventas de cigarrillos, cervezas, vinos, licores y similares en lo corrido del 2016 y Tunja, donde 26 de los 30 entrevistados manifestó que las ventas han sido regulares (17) o muy malas (9).

Tabla 20. Apreciación ventas 2017 según tamaño de establecimiento

\begin{tabular}{|c|c|c|c|c|}
\hline $\begin{array}{l}\text { Cuenta de } 6 \text {. Ven } \\
\text { Etiquetas de fila }\end{array}$ & $\begin{array}{l}017 \text { Etiquet } \overline{\boldsymbol{\top}} \\
\nabla \text { BUENAS }\end{array}$ & MUY MALAS & REGULAR & Total general \\
\hline GRANDE & 2 & 4 & 8 & 14 \\
\hline MEDIANO & 4 & 4 & 10 & 18 \\
\hline PEQUEÑO & 5 & 5 & 16 & 26 \\
\hline Total general & 11 & 13 & 34 & 58 \\
\hline
\end{tabular}

Es evidente que los negocios pequeños son los que se muestran más pesimistas en cuanto a la apreciación de sus ventas en lo corrido de 2017, seguidos por los medianos. Por lo general en lo observado en las entrevistas son los negocios pequeños los que se ven más restringidos en sus ventas porque el horario de cierre limita sus ventas en tanto que los negocios grandes, como autoservicios o supermercados, venden los productos objeto de este estudio en su mayoría para llevar.

Tabla 21. Comparación ventas de 2016 con ventas de 2017 según tamaño del establecimiento

\begin{tabular}{|c|c|c|c|c|}
\hline \multicolumn{5}{|l|}{ Cuenta de 7. M Etiqueta $\bar{\nabla}$} \\
\hline Etiquetas de $\bar{\nabla}$ IGUALES & MEJORES & 5 NO SABE & PEORES & Total general \\
\hline GRANDE & 1 & 1 & 11 & 13 \\
\hline MEDIANO & 3 & & 12 & 15 \\
\hline PEQUEÑO & 2 & 1 & 19 & 22 \\
\hline Total general & 6 & 1 & 42 & 50 \\
\hline
\end{tabular}

Entre los entrevistados que respondieron esta pregunta, una abrumadora mayoría manifestó su pesimismo en lo relativo a la comparación de las ventas de cigarrillos, cervezas, vinos, licores y similares entre 2016 y 2017, pues 42 dijeron que las ventas han sido peores este año que el año pasado, destacándose la proporción de establecimientos pequeños que manifestaron dicho pesimismo.

Tabla 22. Comparación ventas de 2016 con ventas de 2017 por razones sentidas

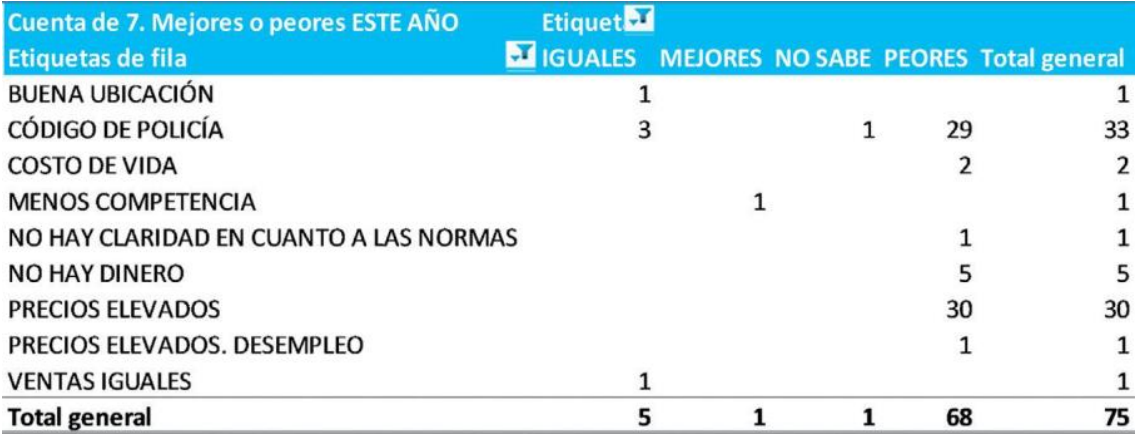


Al ver las razones expresadas según el comportamiento de las ventas en lo corrido de 2017, quienes expresan que han sido peores lo atribuyen principalmente Código de Policía y al alza de los precios. Es preciso hacer la aclaración de que la mayoría de entrevistados asimila el Decreto Municipal que regula el consumo de licores y cigarrillos en sus establecimientos con la entrada en vigencia del Código de Policía, lo cual no es de todo cierto, pues si bien el Código enmarcaría ciertas pautas de comportamiento en el consumo de los mencionados pro-ductos, los decretos Municipales han entrado en vigencia con cierta anterioridad al Código. El alza de los precios también se destaca como una razón importante para señalar que las ventas de 2017 han sido peores que las de 2016.

Tabla 23. Ventas de 2017 (enero a abril) según razones sentidas

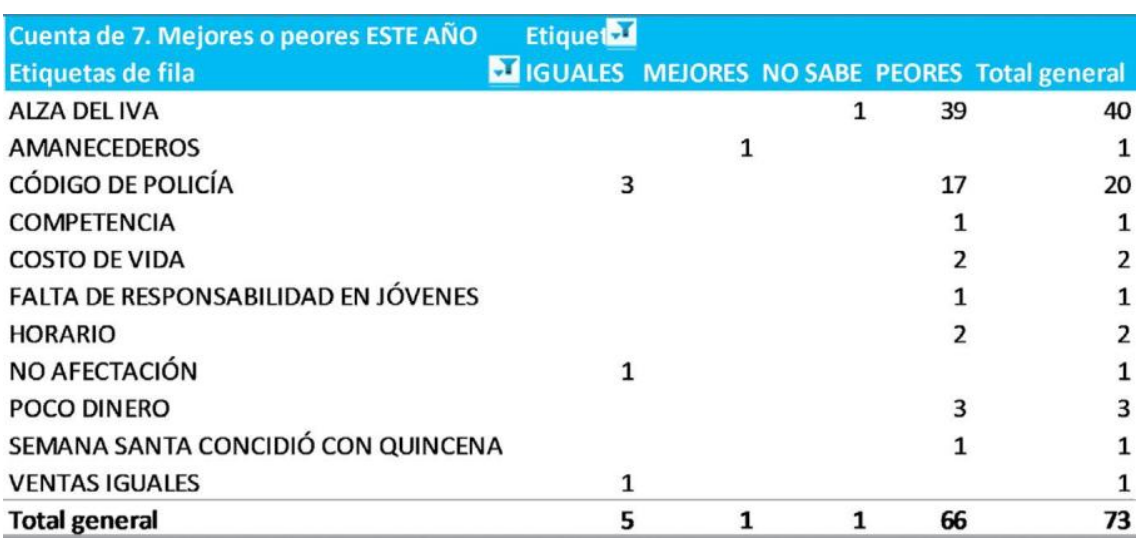

Al observar la tabla se aprecia de manera clara que el alza del IVA seguida por el impacto del Código de Policía son las razones que mayoritariamente expresan los entrevistados para que las ventas del 2017 sean peores que las de 2016. Las demás razones parecen irrelevantes al ver la contundencia de la cifra de entrevistados que señalan al Alza del IVA y al Código de Policía.
Tabla 24. Prospectiva de ventas 2017 según tamaño del establecimiento

\begin{tabular}{lrrrr} 
Cuenta de 13. Prospecti Etiquetas d $\mathbf{\nabla}$ & & & \\
Etiquetas de fila & $\nabla$ GRANDE & MEDIANO & PEQUEÑO & Total general \\
IGUAL & 2 & 1 & & 3 \\
MEJOR & 4 & 4 & 6 & 14 \\
NOSABE & & 1 & & 1 \\
PEOR & 8 & 9 & 16 & 33 \\
\hline Total general & $\mathbf{1 4}$ & $\mathbf{1 5}$ & $\mathbf{2 2}$ & $\mathbf{5 1}$ \\
\hline
\end{tabular}

Total genera

De los 51 entrevistados que respondieron esta pregunta más de la mitad manifiesta que sus ventas de cigarrillos, cervezas, vinos, licores y similares para 2017 serán peores de lo que fueron para 2016, principalmente de los establecimientos pequeños, opinión que comparten, aunque en menor medida, los establecimientos medianos y grandes.

Tabla 25. Prospectiva de ventas 2017 por ciudad

\begin{tabular}{|c|c|c|c|c|c|c|}
\hline $\begin{array}{l}\text { Cuenta de } 13 \text {. Pro } \\
\text { Etiquetas de fila }\end{array}$ & $\begin{array}{l}\text { ven Etiquetas de } \bar{\square} \\
\nabla \text { CHIQUINQUIRÁ }\end{array}$ & MA & IPA : & Aмо & & neral \\
\hline IGUAL & 1 & 4 & 1 & 1 & 3 & 10 \\
\hline MEJOR & 1 & 8 & 3 & 8 & 5 & 25 \\
\hline NOSABE & & & & & 2 & 2 \\
\hline PEOR & 13 & 8 & 11 & 11 & 12 & 55 \\
\hline Total general & 15 & 20 & 15 & 20 & 22 & 92 \\
\hline
\end{tabular}

Indistintamente de la ciudad en la que funcione el establecimiento, la prospectiva de las ventas de cigarrillos, cerve-zas, vinos, licores y similares es bastante pesimista, siendo Chiquinquirá (13 de 15), Sogamoso (11 de 20) y Paipa (11 de

15) donde la proporción de entrevistados manifiesta mayoritariamente que las ventas a final de 2017 serán peores que a final de 2016. 
Tabla 26. Prospectiva de ventas 2017 por tipo de establecimiento

\begin{tabular}{|c|c|c|c|c|c|}
\hline \multicolumn{4}{|c|}{ Cuenta de 13. Prospectii Etiquetas de columna } & \multirow[b]{2}{*}{ NO SABE } & \multirow[b]{2}{*}{ Total general } \\
\hline Etiquetas de fila & IGUAL & MEJOR & PEOR & & \\
\hline ALMACÉN DE CADENA & & & 1 & & 1 \\
\hline AUTOSERVICIO & 1 & & 2 & & 3 \\
\hline BAR, PUB, TABERNA & 6 & 18 & 25 & & 49 \\
\hline CAFETERÍA & & 1 & 1 & & 2 \\
\hline LONCHERÍA & & & 1 & & 1 \\
\hline CIGARRERÍA & 1 & & 1 & 1 & 3 \\
\hline CLUB & & 1 & & & 1 \\
\hline CLUB DE BILLARES & & 1 & 1 & & 2 \\
\hline CLUB DE TEJO & & 1 & & & 1 \\
\hline DEPÓSITO DE CERVEZA & & & 1 & & 1 \\
\hline DISTRIBUIDORA & & & 1 & & 1 \\
\hline \multicolumn{2}{|c|}{ DISTRIBUIDORA DE DULCES } & & 1 & & 1 \\
\hline BILLAR & & & 1 & & 1 \\
\hline LICORERA & & & 2 & & 2 \\
\hline RESTAURANTE & 1 & & & 1 & 2 \\
\hline ROCKOLA Y TIENDA & & & 1 & & 1 \\
\hline SUPERMERCADO & 1 & & & & 1 \\
\hline TIENDA & & 3 & 16 & & 19 \\
\hline Total general & 10 & 25 & 55 & 2 & 92 \\
\hline
\end{tabular}

Los negocios que se muestran más pesimistas en cuanto a la prospectiva de ventas para final de 2017 son los bares, pubs, tabernas y las tiendas, corroborando que son los establecimientos que venden cigarrillos, cervezas, vinos, licores y similares para consumo inmediato los que se muestran más afectados por las medidas ya mencionadas. Una porción menor de los bares, pubs y tabernas manifiestan que las ventas a finales de 2017 serán mejores que en 2016.
Tabla 27. Opinión código de policía por ciudad

\begin{tabular}{|c|c|c|c|c|}
\hline Cuenta de 14. Opinión Código Policía & Etiquetas de col & lumna & & \\
\hline Etiquetas de fila & CHIQUINQUIRÁ & DUITAMA & PAIPA & SOGAM \\
\hline AFECTA LAS VENTAS, ES MALO, PERJUDICIAL & 1 & 1 & & 3 \\
\hline ARBITRARIO, SEVERO, ESTRICTO, RÍGIDO & & & & 7 \\
\hline NO ES TAN BUENO & & & & 1 \\
\hline TIENE COSAS BUENAS PERO... & 10 & 15 & & 2 \\
\hline \multicolumn{5}{|l|}{ EN ALGUNOS ASPECTOS GENERA CONFUSIONES } \\
\hline ES UN ABUSO. GENERA AFECTACIONES & & 1 & & \\
\hline \multicolumn{5}{|l|}{ FALTA RESPETO HACIA EL CIUDADANO. ABUSOS } \\
\hline HAY MUCHAS IRREGULARIDADES & 1 & & & \\
\hline HAY QUE REFORMARLO & & 3 & & \\
\hline MULTAS RESTRINGIDO AL CONSUMIDOR FINAL & & & 1 & 1 \\
\hline NO ES CLARO & & & & \\
\hline
\end{tabular}

Si bien las respuestas a esta pregunta parecen muy variadas la tendencia es hacia que el Código de Policía tiene cosas buenas, pero afecta el consumo, las ventas de cigarrillos, cervezas, vinos, licores y similares y que se presta para abusos de parte de los agentes de policía. Hay que resaltar que poco menos de 10 entrevistados manifiestan no verse afectados por las medidas del Código de Policía, con oposición a la am-plia mayoría que, aunque le reconoce sus virtudes, también manifiesta que tiene cosas que se pueden mejorar, sobre todo las que los afectan en sus ventas. Si bien la respuesta "tiene cosas buenas, pero" obtiene una alta puntuación, hay que resaltar que entre los peros se destacan contrastes como que el código tiene cosas buenas pero afecta las ventas, que hay que reformarlo o que se presta para abusos. Esto reve-la que el Código es una medida con buena aceptación por parte de los comerciantes, aunque señalan también que los perjudica de una u otra manera, sea en cuanto a las ventas o porque se presta para abusos. 
Tabla 28. Opinión código de policía por prospectiva de ventas 2017

\begin{tabular}{|c|c|c|c|c|}
\hline Cuenta de 14. Opinión Código Policía & Etique 7 & & & \\
\hline Etiquetas de fila & VIGUAL & & OR T & \\
\hline AFECTA & & & 1 & 1 \\
\hline AFECTA POR HORARIO. & & 1 & 3 & 4 \\
\hline AFECTA VENTAS & & & 1 & 1 \\
\hline ARBITRARIO & & & 1 & 1 \\
\hline DEMASIAFO RESTRICTIVO & & & 1 & 1 \\
\hline EN ALGUNOS ASPECTOS GENERA CONFUSIONES & 1 & 1 & & 1 \\
\hline ES MALO PARA LAS VENTAS & & & 1 & 1 \\
\hline ES UN ABUSO. GENERA AFECTACIONES & & 1 & & 1 \\
\hline ESTÁ MAL & & & 1 & 1 \\
\hline HA SIDO PERJUDICIAL & & & 1 & 1 \\
\hline HAY MUCHAS IRREGULARIDADES & 1 & 1 & & 1 \\
\hline HAY QUE REFORMARLO & 1 & 1 & 4 & 6 \\
\hline MULTAS RESTRINGIDO AL CONSUMIDOR FINAL & & & 1 & 1 \\
\hline MUY ARBITRARIO & & 1 & 2 & 3 \\
\hline MUY ESTRICTO & & & 3 & 3 \\
\hline MUY MALO. AFECTA VENTAS & & & 2 & 2 \\
\hline MUY RESTRICTIVO & & 1 & & 1 \\
\hline MUY SEVERO & & & 1 & 1 \\
\hline NO ES CLARO & & & 1 & 1 \\
\hline NO ME AFECTA & 1 & 3 & 4 & 8 \\
\hline NO ME PARECE & & 1 & & 1 \\
\hline NO SE VEAÚN & & & 1 & 1 \\
\hline NO TAN BUENO & & 2 & & 2 \\
\hline PERJUDICA A LOS BARES & & & 1 & 1 \\
\hline TIENE ARTÍCULOS QUE NO SON COHERENTES & & & 1 & 1 \\
\hline TIENE COSAS BUENAS & 1 & 1 & 2 & 4 \\
\hline TIENE COSAS BUENAS Y OTRAS COSAS MALAS & 2 & 2 & 5 & 9 \\
\hline TIENE COSAS BUENAS. & & 1 & & 1 \\
\hline TIENE COSAS BUENAS. AFECTA VENTAS & 1 & 4 & 4 & 9 \\
\hline TIENE COSAS BUENAS. AFECTA VENTAS & & & 2 & 2 \\
\hline TIENE COSAS BUENAS. AFECTA VENTAS POR HORARIO & & 1 & 2 & 3 \\
\hline TIENE COSAS BUENAS. HACE FALTA REFORMA & 1 & 2 & 3 & 6 \\
\hline TIENE COSAS BUENAS. NO ES IGUAL PARA TODOS & & & 2 & 2 \\
\hline TIENE COSAS POSITIVAS & & & 2 & 2 \\
\hline TIENE COSAS POSITVAS Y OTRAS COSAS MALAS & & 1 & & 1 \\
\hline TIENE COSAS POSITIVAS. ABUSOS & 1 & 1 & 2 & 4 \\
\hline UN POCO RÍGIDO. NO BAJA CONSUMO, CAMBIA HÁBITOS, TODO SON MULTAS & & 1 & & 1 \\
\hline Total general & 10 & 25 & 55 & 90 \\
\hline
\end{tabular}

iguiendo con la tendencia que muestra pesimismo en relativo a las ventas al finalizar el presente año, la amplia mayoría de quienes se ven afectados por el Código de Policía dicen que las ventas serán peores que en 2016.
Tabla 29. Prospectiva de ventas 2017 por aumento de precios

\begin{tabular}{lrrrr} 
Cuenta de 13. Pros Etique & & & \\
Etiquetas de fila & MUCHO & NADA & POCO & Total general \\
IGUAL & 3 & 1 & 6 & 10 \\
MEJOR & 9 & 2 & 14 & 25 \\
PEOR & 30 & 8 & 17 & 55 \\
\hline Total general & $\mathbf{4 2}$ & $\mathbf{1 1}$ & $\mathbf{3 7}$ & $\mathbf{9 0}$ \\
\hline
\end{tabular}

Al preguntar por la proporción en que tuvieron que aumentar los precios debido a las medidas tributarias del año pasado, quienes dicen que tuvieron que aumentar los precios mucho son quienes mayoritariamente se muestran pesimistas a la hora de pensar en las ventas a final de 2017.

Tabla 30. Aumento de precios por ciudades

\begin{tabular}{|c|c|c|c|c|c|}
\hline \multicolumn{6}{|c|}{$\begin{array}{l}\text { Cuenta de Ciudad Etiquetas d } \\
\text { Etiquetas de fila } ~\end{array}$} \\
\hline CHIQUINQUIRÁ & 15 & & & & 15 \\
\hline DUITAMA & 3 & 3 & 14 & & 20 \\
\hline PAIPA & 10 & 3 & 2 & & 15 \\
\hline SOGAMOSO & 13 & & 7 & & 20 \\
\hline TUNJA & 2 & 7 & 18 & 3 & 30 \\
\hline Total general & 43 & 13 & 41 & 3 & 100 \\
\hline
\end{tabular}

Por ciudades el aumento de precios presenta un comportamiento variado. En primer lugar Duitama fue la ciudad donde la mayoría de los entrevistados manifestó haber subido poco los precios, en tanto que en Chiquinquirá y Paipa la abrumado-ra mayoría expresó haber tenido que subir mucho los precios.

Tabla 31. Influencia de los festivos por ciudades

\begin{tabular}{lrrrr}
\hline Cuenta de Ciudad & Etiquetas & & & \\
Etiquetas de fila & INDIFERENTENEGATIVA & POSITIVA & Total general \\
CHIQUINQUIRÁ & 4 & 2 & 9 & 15 \\
DUITAMA & 4 & 2 & 14 & 20 \\
PAIPA & 1 & 8 & 6 & 15 \\
SOGAMOSO & 5 & 6 & 9 & 20 \\
TUNJA & 12 & 12 & 6 & 30 \\
\hline Total general & $\mathbf{2 6}$ & $\mathbf{3 0}$ & $\mathbf{4 4}$ & $\mathbf{1 0 0}$ \\
\hline
\end{tabular}


Los Municipios entrevistados donde se muestra una influen-cia positiva de los festivos en el consumo de cigarrillos, cer-vezas, vinos, licores y similares son Duitama y Chiquinquirá a diferencia de unja donde fueron más quienes expresaron una influencia negativa de los fines de semana con festivo en el consumo de tales productos.

Tabla 32. Aumento de precios según tipo de establecimiento

\begin{tabular}{|c|c|c|c|c|}
\hline $\begin{array}{l}\text { Cuenta de } 16 \text {. Aumento precios } \\
\text { Etiquetas de fila }\end{array}$ & $\begin{array}{l}\text { of Etiquet } \sqrt{\bar{x}} \\
\nabla \text { MUсHO }\end{array}$ & NADA & $\mathrm{CO}$ & \\
\hline ALMACÉN DE CADENA & 1 & & & 1 \\
\hline AUTOSERVICIO & 3 & & & 3 \\
\hline BAR & 11 & 4 & 7 & 22 \\
\hline BAR NNOCTURNO & 1 & & & 1 \\
\hline BILLAR & 1 & & & 1 \\
\hline CAFÉ BAR & 1 & & & 1 \\
\hline CAFÉ BAR & 1 & 3 & 13 & 17 \\
\hline CAFETERÍA & & & 3 & 3 \\
\hline CAFETERÍA BAR & 1 & & & 1 \\
\hline CIGARRERÍA & 2 & 1 & 3 & 6 \\
\hline CLUB & & 1 & & 1 \\
\hline CLUB DE BILLARES & & 1 & 1 & 2 \\
\hline CLUB DE TEJO & & & 1 & 1 \\
\hline DEPÓSITO DE CERVEZA & 1 & & & 1 \\
\hline DISTRIBUIDORA & 1 & & & 1 \\
\hline DISTRIBUIDORA DE DULCES & 1 & & & 1 \\
\hline KARAOKE BAR & 1 & & & 1 \\
\hline LICORERA & 1 & & 1 & 2 \\
\hline LONCHERÍA & 1 & & & 1 \\
\hline RESTAURANTE & & & 1 & 1 \\
\hline RESTAURANTE BAR & 1 & & 1 & 2 \\
\hline RESTAURANTE CAFÉ BAR & 1 & & & 1 \\
\hline RESTAURANTE CERVECERÍA PUB & & & 1 & 1 \\
\hline RESTAURANTE PUB & & & 1 & 1 \\
\hline ROCKOLA Y TIENDA & 1 & & & 1 \\
\hline SUPERMERCADO & 1 & & & 1 \\
\hline TABERNA & 1 & & & 1 \\
\hline TIENDA & 10 & 3 & 7 & 20 \\
\hline TIENDA BAR & & & 1 & 1 \\
\hline Total general & 43 & 13 & 41 & 97 \\
\hline
\end{tabular}

Los bares y tiendas son los establecimientos que más manifestaron haber tenido que aumentar mucho los precios.
Tabla 33. Ayuda que puede brindar la gobernación por ciudades

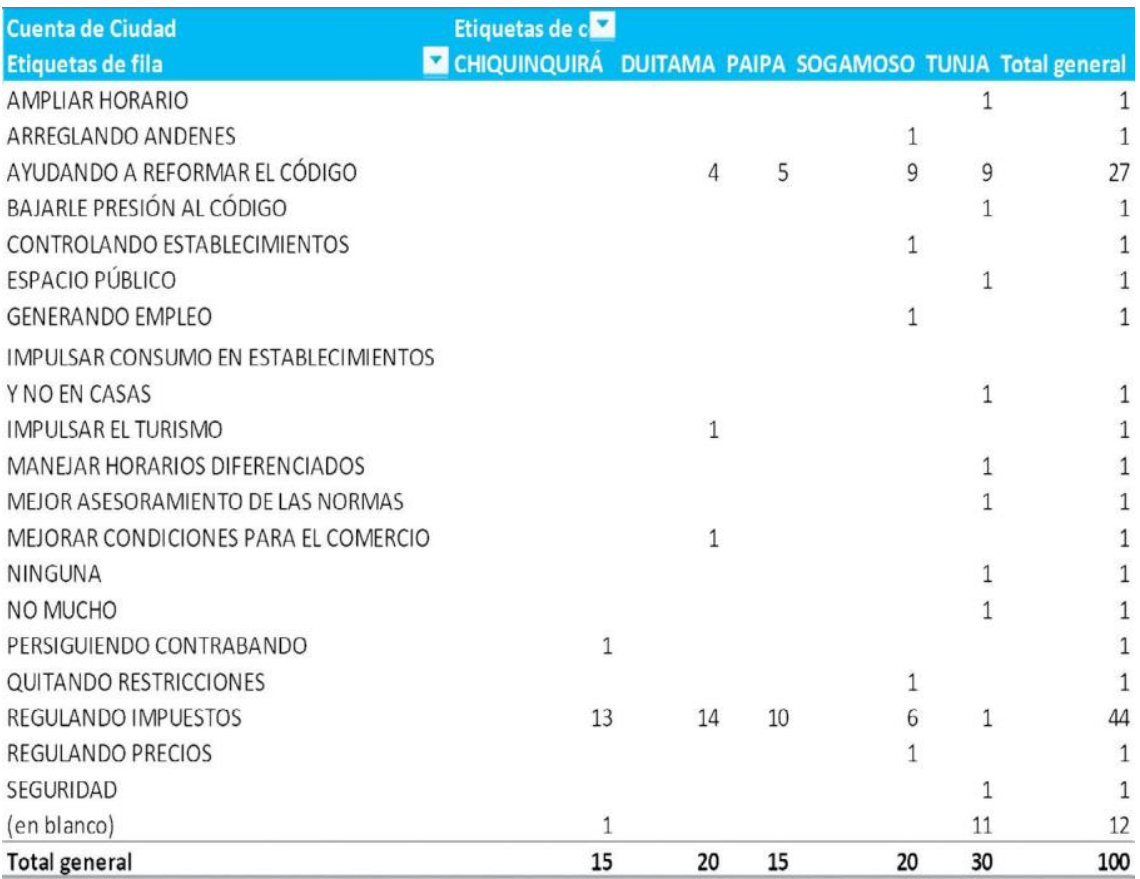

Esta tabla evidencia el nivel de desconocimiento que tienen los propietarios de establecimientos en lo relativo a las funcio-nes de la Secretaría de Hacienda o la Gobernación en cuanto a la normatividad, pues una gran proporción piensa que una manera de ayudar a que se mejoren las ventas de cigarrillos, cervezas, vinos, licores y similares es ayudando a reformar el Código de Policía. Una proporción aún mayor, manifiesta que una forma de ayudarlos es regulando o bajando los impues-tos, sobretodo el IVA. 
Tabla 34. Ventas de 2017 (enero a abril) según razones sentidas

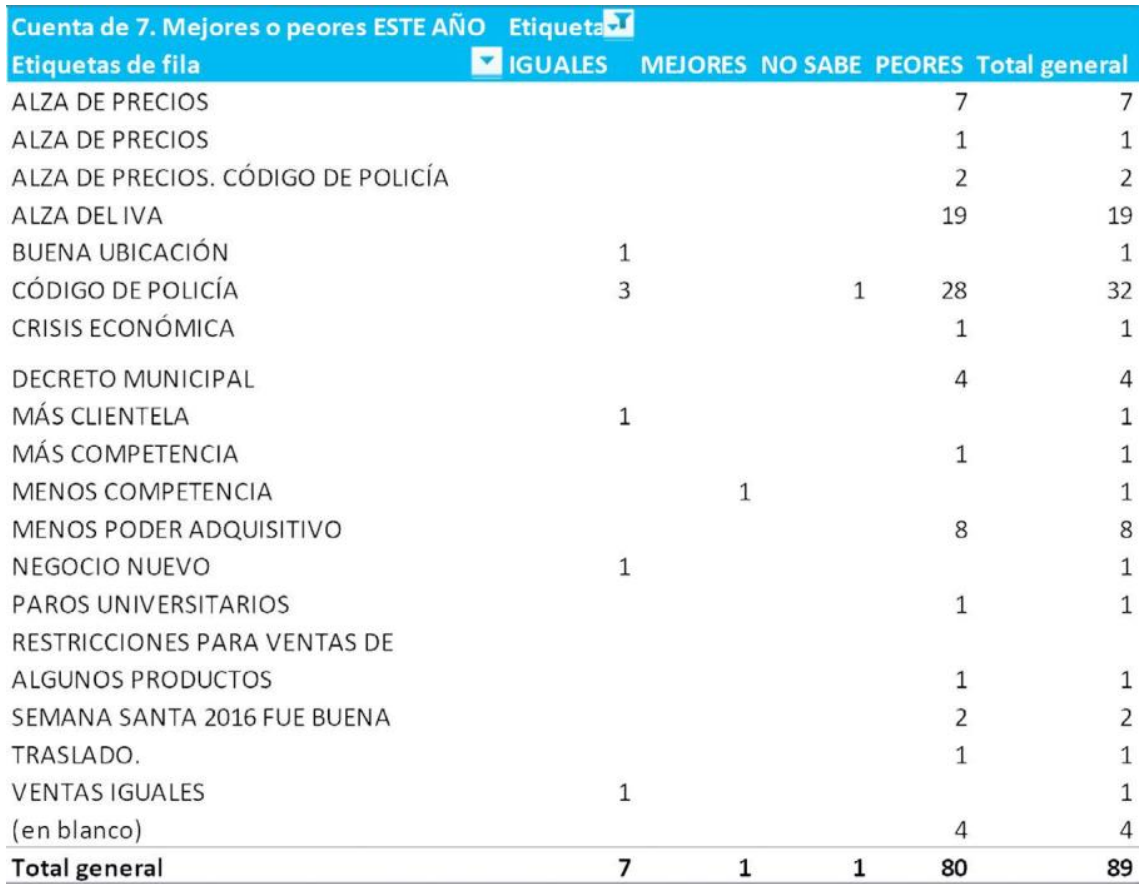

Quienes manifiestan que las ventas de cigarrillos, cervezas, vinos, licores y similares han sido peores en 2017 que en 2016 expresan como razones de esto el alza del IVA y el Código de Policía, seguido por la caída en el poder adquisitivo de las personas.

Tabla 35. Aumento de precios según ayuda que puede brindar la gobernación. (Más puntuadas: regulando impuestos y ayudando a reformar el

\begin{tabular}{lrrrr}
\multicolumn{1}{c}{$\begin{array}{c}\text { código) } \\
\text { Etiquetas de fila }\end{array}$} & \multicolumn{1}{c}{ MUCHO } & NADA & POCO & Total general \\
AYUDANDO A REFORMAR EL CÓDIGO & 8 & 5 & 14 & 27 \\
REGULANDO IMPUESTOS & 26 & 4 & 14 & 44 \\
\hline Total general & 34 & $\mathbf{9}$ & $\mathbf{2 8}$ & $\mathbf{7 1}$ \\
\hline
\end{tabular}

Los entrevistados que manifiestan haberle subido mucho a los precios son los mismos que mayoritariamente piensan que la Gobernación les puede ayudar regulando los impuestos para mejorar las ventas de cigarrillos, cervezas, vinos, licores y similares.

Tabla 36 Razones de las ventas de 2017 (p. 8) por ciudades

\begin{tabular}{|c|c|c|c|c|c|c|}
\hline $\begin{array}{l}\text { Cuenta de Ciudad } \\
\text { tstituthate fil }\end{array}$ & Etiquetas de & & A A A & & & \\
\hline $\begin{array}{l}\text { Etiquetas de fila } \\
\text { ALZA DE PRECIOS }\end{array}$ & 6 & & & 1 & & eneral 7 \\
\hline ALZA DE PRECIOS & 1 & & & & & \\
\hline ALZA DE PRECIOS. CÓDIGO DE POUCIAA & 2 & & & & & \\
\hline ALZA DEL IVA & & 11 & & 4 & 3 & 19 \\
\hline BUENA UBICACIÓN & & & & & & \\
\hline CLIENTELA. UBICACIÓN & & & & & 1 & 1 \\
\hline CÓDIGO DE POLCÍA & & 7 & 1 & 5 & 8 & 32 \\
\hline CRISIS ECONÓMICA & & & & & & 1 \\
\hline DECRETO MUNICIPAL & & & & & 6 & 6 \\
\hline LO FUERTE ES EL LRESTAURANTE & & & & & 1 & 1 \\
\hline MÁS CLIENTELA & & & & & 1 & 1 \\
\hline MÁS COMPETENCIA & 1 & & & & & 1 \\
\hline MENOS COMPETENCIA & & & & 1 & & 1 \\
\hline MENOS PODER ADQUISITIVO & 4 & & & 3 & 1 & 8 \\
\hline NEGOCIO NUEVO & & & & & 1 & \\
\hline PAROS UNIVERSITARIOS & & & & 1 & & \\
\hline RESTRICCIONES PARA VENTAS DE ALGUNOS PRODUCTOS & & 1 & & & & \\
\hline SEMANA SANTA 2016 FUE BUENA & & 1 & & & 1 & 2 \\
\hline TRASLADO. & & & & & 1 & \\
\hline VENTAS IGUALES & & & & 1 & & \\
\hline (en blanco) & 1 & & & 4 & 6 & 11 \\
\hline Total general & 15 & 20 & 1 & 20 & 30 & 100 \\
\hline
\end{tabular}

Las razones que más puntúan por parte de los entrevistados para explicar por qué las ventas de cigarrillos, cervezas, vinos, licores y similares se comportan como lo hacen son el Código de Policía y las alzas del IVA y de precios.

\section{CONCLUSIONES}

Los comerciantes de cigarrillos, cervezas, licores, vinos y similares manifiestan haberse visto afectados por la entada en vigencia del Código de Policía. Si bien al observar detalladamente las razones expuestas por ellos, se evidencia que la mayor afectación manifiesta proviene de los Decretos Municipales que regulan el consumo de licor y los horarios de venta.

La amplia mayoría de los comerciantes entrevistados manifiesta que las ventas del primer trimestre de 2016 fueron mejores que las del mismo período de 2017. 
La mayoría de los comerciantes entrevistados señalan que las ventas a finales de 2017 serán peores que las de 2016. Como causa principal señalan la entrada en vigencia del Código y las alzas en los precios.

Los entrevistados expresan que la Gobernación podría colaborar con el incremento de las ventas si les ayuda a reformar el Código o regulando los impuestos. De esta conclusión se puede extraer una recomendación en el sentido de la pedagogía y la formación tributaria plasmada en el Plan de Desarrollo Departamental Creemos en Boyacá, 2016 - 2019. En la medida en que se desarrollen programas sociales de formación tributaria, la ciudadanía en general conocerá de mejor manera el sistema tributario departamental, su funcionamiento y las maneras en que puede vincularse para mejorar el recaudo de los tributos en el Departamento.

\section{RECOMENDACIONES}

Se hace imperativo realizar una segunda medición de la misma problemática en el mismo período de tiempo en el año 2018 con el fin de tener los insumos necesarios para realizar una comparación y determinar si el impacto en las vetas de cervezas, vinos, licores y cigarrillos se sostiene o tiende a variar.

Bueno sería que este tipo de medias se tomen teniendo en cuenta los puntos de vistas de todas las partes implicadas para que los efectos negativos sean mínimos.

El nivel de conocimiento de la normatividad que afecta los precios de las cervezas, vinos, licores y cigarrillos es una variable que podría ser tenida en cuenta en futuros análisis. Esto enfatiza la importancia que debe tener la socialización de la normatividad entre la población del Departamento de Boyacá.

\section{REFERENCIAS}

Babbie, E. (s.f). Fundamentos de la investigación social. Universidad de Chapman.

Congreso de la república de Colombia. (s.f.). Ley. Ley 1816 de 2016.

Congreso de la república de Colombia. (s.f.). Ley. Ley 1918 de 2016. Bogotá, Colombia.

Plan de desarrollo Departamental de Boyacá. (2016-2019). Creemos en Boyacá. Tunja. 


\section{BIOGRAFÍA DE LOS AUTORES}

Lizeth Viviana Lesmes Ortiz. Administradora de Empresas, Especialista en Fianzas, Estudiante de maestría en Administración de Empresas con Especialidad en Gerencia de Proyectos. Joven investigador Colciencias. Docente Investigadora con proyectos ejecutados como investigador principal en la línea de Economía, empresa y región y empresarismo, en temas como turismo, marketing de ciudades o territorial y políticas públicas de emprendimiento. Autora de capítulos de libro y árbitro evaluador de libros de la red de competitividad, innovación y desarrollo sustentable de la Universidad de Colima México.

Juan Medina Roa Comunicador Social, con estudios en curso de Maestría en Historia de la Universidad Pedagógica y Tecnológica de Colombia - e investigador del Grupo de Ciencias Administrativas y Contables de la Universidad Santo Tomás. Director del Instituto de Estudios Socio-históricos

Fray Alonso de Zamora, con experiencia en las líneas de trabajo asociadas a historia, cultura, patrimonio. Ha Publicado La Huella Salesiana con Leonardo Gómez Hernández, Las Hermanas de la Caridad de Santa Ana, Una Mirada A Güican, 
Tunja desde 1900, 100 años de la Cámara de Comercio de Tunja y varios artículos de historia y otros temas. Es Miembro de número de la Academia Boyacense de Historia, de la Academia Eclesiástica de Boyacá y de la Academia Nariñista de Boyacá.

Nataly Yohana Callejas Rodriguez Contadora Pública, Administradora de Empresas, Magister en Administración de Empresas en la Universidad Arturo Prat de Chile - e investigadora del Grupo de Ciencias Administrativas y Contables de la Universidad Santo Tomás. Con experiencia en las líneas de trabajo asociadas a contabilidad ambiental y economía, empresa y región.

Luri Suárez Pineda Magíster en Administración de la Universidad Nacional de Colombia, Especialista en Proyectos de Desarrollo de la ESAP, Administradora de Empresas de la UPTC. Docente de la Universidad Santo Tomás, Seccional Tunja. Grupo de investigación en Ciencias Administrativas y Contables. Con publicaciones relacionadas como "Comportamiento del sec-tor lechero en el departamento de Boyacá: una aproximación desde la metáfora biológica" en la revista Punto De Vista del Politécnico Grancolombiano.

Marlén Suárez Pineda Estudiante de doctorado en Administración de la Universidad Autónoma de Querétaro, Magíster en Administración del ITESM-UNAB, Especialista en Administración de Negocios Internacionales de la EAN, Administradora de Empresas de la Universidad Pedagógica y Tecnológica de Colombia UPTC. Docente de la UPTC. Grupo de investigación IDEAS. Ha desarrollado publicaciones relacionados con el sector papero, quinua y procesos de innovación microempresarial en revistas regionales y nacionales.

Nancy Patricia García Pacheco Profesional en Publicidad y mercadeo, Especialista en gerencia de proyectos, Magister en educación, PhD en Administración de Empresas.
Docente- Investigadora Universidad santo Tomás, seccional Tunja. Ha desarrollado publicaciones en revistas internacionales como Horizonte empresarial en temas de marketing, publicidad y financiamiento microempresarial.

Elina Ulloa Sáenz Abogada de la Universidad Santo Tomás seccional Tunja, Especialista en Derecho Constitucional y en Contratación Estatal, Magister en Argumentación Jurídica de la Universidad de Alicante. (Alicante- España); con experiencia laboral en el sector público en Hacienda Pública, Contratación Estatal, Desarrollo Humana, asistencia legislativa y en asuntos de seguridad y convivencia ciudadana.

Carlos Andrés Aranda Camacho Abogado de la Universidad Santo Tomás seccional Tunja, Especialista en Derecho Administrativo, Magister en Derecho Administrativo de la Universidad Santo Tomás seccional Tunja, Docente Universitario, miembro del grupo de Investigación en Ciencias Administrativas y Contables de la Universidad Santo Tomás seccional.

Luis Rodrigo Ramírez Ahumada Sociólogo de la Universidad Nacional de Colombia, experto en diseño metodológico de la investigación social. Ha participado en múltiples investigaciones sociológicas en diversos centros de pensamiento, especialmente en salud, cultura ciudadana y política 


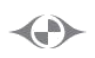

Este libro se terminó de imprimir en diciembre de 2018 en los talleres de Editorial Jotamar Ltda. Tunja, Boyacá, Colombia. 
Esta obra es el producto del esfuerzo de los investigadores que conforman el grupo de investigación en ciencias económicas, administrativas y contables de la Universidad Santo Tomás Seccional Tunja.

El propósito de este libro es difundir las investigaciones realizadas en el estudio de las líneas de investigación del grupo, asociadas a problemáticas y necesidades del entorno. En este caso, investigaciones delimitadas al Departamento de Boyacá, en busca de contribuir a mejorar las condiciones del sector económico y empresarial de la región en temas como el sector agroindustrial, turístico, organizacional de las Mi Pymes y la gestión pública articulado con las tendencias que propone la academia en las áreas relacionadas.

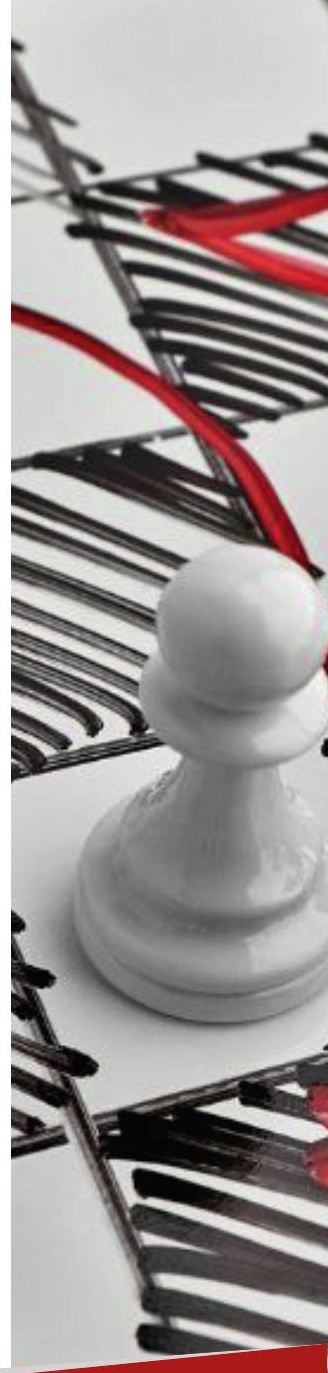

